GLASNIK MATEMATIČKI

Vol. 38(58)(2003), $253-261$

\title{
AN EQUATION RELATED TO CENTRALIZERS IN SEMIPRIME RINGS
}

\author{
Joso Vukman and IRena Kosi-Ulbl \\ University of Maribor, Slovenia
}

\begin{abstract}
The main result: Let $R$ be a 2-torsion free semiprime ring with extended centroid $C$ and let $T: R \rightarrow R$ be an additive mapping. Suppose that $3 T(x y x)=T(x) y x+x T(y) x+x y T(x)$ holds for all $x, y \in R$. Then there exists an element $\lambda \in C$ such that $T(x)=\lambda x$ for all $x \in R$.
\end{abstract}

This research has been motivated by the work of Brešar [4] and Zalar [8]. Throughout, $R$ will represent an associative ring with center $Z(R)$. A ring $R$ is $n$-torsion free, where $n>1$ is an integer, in case $n x=0, x \in R$ implies $x=0$. As usual the commutator $x y-y x$ will be denoted by $[x, y]$. We shall use basis commutator identities $[x y, z]=[x, z] y+x[y, z]$ and $[x, y z]=[x, y] z+y[x, z]$. Recall that $R$ is prime if $a R b=(0)$ implies $a=0$ or $b=0$, and is semiprime if $a R a=(0)$ implies $a=0$. An additive mapping $D: R \rightarrow R$ is called a derivation if $D(x y)=D(x) y+x D(y)$ holds for all pairs $x, y \in R$ and is called a Jordan derivation in case $D\left(x^{2}\right)=D(x) x+x D(x)$ is fulfilled for all $x \in R$. A derivation $D$ is inner in case there exists $a \in R$, such that $D(x)=[a, x]$ holds for all $x \in R$. Every derivation is a Jordan derivation. The converse is in general not true. A classical result of Herstein [6] asserts that any Jordan derivation on a 2-torsion free prime ring is a derivation (see [2] for an alternative proof). Cusack [5] generalized Herstein's result on 2torsion free semiprime rings (see [3] for an alternative proof). We follow Zalar [8] and call an additive mapping $T: R \rightarrow R$ a left (right) centralizer in case $T(x y)=T(x) y \quad(T(x y)=x T(y))$ holds for all $x, y \in R$. This concept appears naturally in $C^{*}$-algebras. In ring theory it is more common to work with module homomorphisms. Ring theorists would write that $T: R_{R} \rightarrow R_{R}$ is

2000 Mathematics Subject Classification. 16N60, 39B05.

Key words and phrases. Prime ring, semiprime ring, derivation, Jordan derivation, Jordan triple derivation, left (right) centralizer, left (right) Jordan centralizer, centralizer.

This research has been supported by the Research Council of Slovenia. 
a homomorphism of a ring module $R$ into itself. For a semiprime ring $R$ all such homomorphisms are of the form $T(x)=q x$ for all $x \in R$, where $q$ is an element of Martindale right ring of quotients $Q_{r}$ ( see Chapter 2 in [1]). Similarly, we call an additive mapping $T: R \rightarrow R$ a right centralizer in case $T(x y)=x T(y)$ holds for all $x, y \in R$. In case $T: R \rightarrow R$ is left and right centralizer, where $R$ is a semiprime ring with extended centroid $C$, then there exists an element $\lambda \in C$ such that $T(x)=\lambda x$ for all $x \in R$ (see Theorem 2.3.2. in [1]). An additive mapping $T: R \rightarrow R$ is called a left (right) Jordan centralizer in case $T\left(x^{2}\right)=T(x) x \quad\left(T\left(x^{2}\right)=x T(x)\right)$ holds for all $x \in R$. Following ideas from [3] Zalar [8] has proved that any left (right) Jordan centralizer on a 2-torsion free semiprime ring is a left (right) centralizer. An additive mapping $D: R \rightarrow R$, where $R$ is an arbitrary ring, is a Jordan triple derivation in case $D(x y x)=D(x) y x+x D(y) x+x y D(x)$ holds for all pairs $x, y \in R$. One can easily prove that any Jordan derivation is a Jordan triple derivation (see [2]). Brešar [4] has proved that any Jordan triple derivation on a 2-torsion free semiprime ring is a derivation. It is our aim in this paper to prove the result below which was inspired by the result we have just mentioned. Our methods differs from those used in [4]. The proof is, as we shall see, rather long, but it is elementary in the sense that it requires no specific knowledge concerning semiprime rings in order to follow the proof.

TheOREM 1. Let $R$ be a 2-torsion free semiprime ring and let $T: R \rightarrow R$ be an additive mapping. Suppose that

$$
3 T(x y x)=T(x) y x+x T(y) x+x y T(x)
$$

holds for all $x, y \in R$. Then there exists an element $\lambda \in C$ such that $T(x)=$ $\lambda x$ for all $x \in R$.

For the proof of the theorem above we shall need the following lemma and a theorem.

Lemma 2 ([7, Lemma 1]). Let $R$ be a semiprime ring. Suppose that the relation $a x b+b x c=0$ holds for all $x \in R$ and some $a, b, c \in R$. In this case $(a+c) x b=0$ is satisfied for all $x \in R$.

Theorem 3 ([8, Proposition 1.4]). Let $R$ be a 2-torsion free semiprime ring and let $T: R \rightarrow R$ be an additive mapping. Suppose that $T\left(x^{2}\right)=$ $T(x) x \quad\left(T\left(x^{2}\right)=x T(x)\right)$ holds for all $x \in R$. In this case $T$ is a left (right) centralizer.

Proof of Theorem 1. Putting $x+z$ for $x$ in the relation below (linearization)

$$
3 T(x y x)=T(x) y x+x T(y) x+x y T(x), \quad x, y \in R
$$


we obtain

$$
\begin{aligned}
3 T(x y z+z y x)= & T(x) y z+T(z) y x+x T(y) z+z T(y) x \\
& +x y T(z)+z y T(x), \quad x, y, z \in R .
\end{aligned}
$$

For $z=x^{2}$ the relation (2) gives

$$
\begin{aligned}
3 T\left(x y x^{2}+x^{2} y x\right)= & T(x) y x^{2}+T\left(x^{2}\right) y x+x T(y) x^{2}+x^{2} T(y) x \\
& +x y T\left(x^{2}\right)+x^{2} y T(x), \quad x, y \in R .
\end{aligned}
$$

On the other hand substitution $x y+y x$ for $y$ in (1) gives

$$
\begin{aligned}
3 T\left(x y x^{2}+x^{2} y x\right)= & T(x)\left(x y x+y x^{2}\right)+x T(x y+y x) x \\
& +\left(x^{2} y+x y x\right) T(x), \quad x, y \in R .
\end{aligned}
$$

Subtracting (4) from (3) we obtain

(5) $A(x) y x+x y B(x)+x(T(y) x+x T(y)-T(x y+y x)) x=0, \quad x, y \in R$, where $A(x)$ and $B(x)$ stands for $T\left(x^{2}\right)-T(x) x$ and $T\left(x^{2}\right)-x T(x)$, respectively.

Putting $y=x$ and $z=y$ in (2) we obtain

$$
\begin{aligned}
3 T\left(x^{2} y+y x^{2}\right)= & T(x) x y+T(y) x^{2}+x T(x) y \\
& +y T(x) x+x^{2} T(y)+y x T(x), \quad x, y \in R .
\end{aligned}
$$

Putting $3 x$ for $x$ and $z=x^{3}$ in (2) and using (1) we obtain

$$
\begin{aligned}
9 T\left(x y x^{3}+x^{3} y x\right)= & 3 T(x) y x^{3}+3 T\left(x^{3}\right) y x+3 x T(y) x^{3}+3 x^{3} T(y) x \\
& +3 x y T\left(x^{3}\right)+3 x^{3} y T(x) \\
= & 3 T(x) y x^{3}+T(x) x^{2} y x+x T(x) x y x+x^{2} T(x) y x \\
& +3 x T(y) x^{3}+3 x^{3} T(y) x+x y T(x) x^{2}+x y x T(x) x \\
& +x y x^{2} T(x)+3 x^{3} y T(x), \quad x, y \in R .
\end{aligned}
$$

Putting $3\left(x^{2} y+y x^{2}\right)$ for $y$ in (1) and using (6) we obtain

$$
\begin{aligned}
9 T\left(x y x^{3}+x^{3} y x\right)= & 3 T(x)\left(x^{2} y+y x^{2}\right) x+3 x T\left(x^{2} y+y x^{2}\right) x \\
& +3 x\left(x^{2} y+y x^{2}\right) T(x) \\
= & 3 T(x)\left(x^{2} y+y x^{2}\right) x+x\left(T(x) x y+T(y) x^{2}\right. \\
& \left.+x T(x) y+y T(x) x+x^{2} T(y)+y x T(x)\right) x \\
& +3 x\left(x^{2} y+y x^{2}\right) T(x) \\
= & 3 T(x) x^{2} y x+3 T(x) y x^{3}+x T(x) x y x+x T(y) x^{3} \\
& +x^{2} T(x) y x+x y T(x) x^{2}+x^{3} T(y) x+x y T(x) x \\
& +3 x^{3} y T(x)+3 x y x^{2} T(x), \quad x, y \in R .
\end{aligned}
$$

Subtracting (8) from (7) we obtain

$$
T(x) x^{2} y x+x y x^{2} T(x)-x^{3} T(y) x-x T(y) x^{3}=0, \quad x, y \in R .
$$


Putting $3 x y x$ for $y$ in (6) we obtain

$$
\begin{aligned}
9 T\left(x^{3} y x+x y x^{3}\right)= & 3 T(x) x^{2} y x+T(x) y x^{3}+x T(y) x^{3}+x y T(x) x^{2} \\
& +3 x T(x) x y x+3 x y x T(x) x+x^{2} T(x) y x \\
& +x^{3} T(y) x+x^{3} y T(x)+3 x y x^{2} T(x), \quad x, y \in R .
\end{aligned}
$$

On the other hand we obtain putting $z=3 x^{3}$ in (2)

$$
\begin{aligned}
9 T\left(x^{3} y x+x y x^{3}\right)= & 3 T(x) y x^{3}+T(x) x^{2} y x+x T(x) x y x \\
& +x^{2} T(x) y x+3 x T(y) x^{3}+3 x^{3} T(y) x+x y T(x) x^{2} \\
& +x y x T(x) x+x y x^{2} T(x)+3 x^{3} y T(x), \quad x, y \in R .
\end{aligned}
$$

Comparing (10) and (11) we arrive at

$$
\begin{aligned}
& T(x) y x^{3}-T(x) x^{2} y x+x T(y) x^{3}-x T(x) x y x-x y x^{2} T(x) \\
& +x^{3} y T(x)-x y x T(x) x+x^{3} T(y) x=0, \quad x, y \in R .
\end{aligned}
$$

From (9) and (12) one obtains

$$
T(x) y x^{3}-x T(x) x y x+x^{3} y T(x)-x y x T(x) x=0, \quad x, y \in R .
$$

Putting $y x$ for $y$ in the above relation we obtain

$$
T(x) y x^{4}-x T(x) x y x^{2}+x^{3} y x T(x)-x y x^{2} T(x) x=0, \quad x, y \in R .
$$

On the other hand right multiplication of (13) by $x$ gives

$$
T(x) y x^{4}-x T(x) x y x^{2}+x^{3} y T(x) x-x y x T(x) x^{2}=0, \quad x, y \in R .
$$

Subtracting (15) from (14) one obtains

$$
x^{3} y[T(x), x]-x y x[T(x), x] x=0, \quad x, y \in R .
$$

From (16) one obtains

$$
\left[T(x), x^{3}\right] y[T(x), x]-[T(x), x] y x[T(x), x] x=0, \quad x, y \in R .
$$

Now using Lemma we obtain

$$
\left(\left[T(x), x^{3}\right]-x[T(x), x] x\right) y[T(x), x]=0, \quad x, y \in R,
$$

which reduces to

$$
\left([T(x), x] x^{2}+x^{2}[T(x), x]\right) y[T(x), x]=0, \quad x, y \in R .
$$

From the above relation one obtains

$$
\left([T(x), x] x^{2}+x^{2}[T(x), x]\right) y\left([T(x), x] x^{2}+x^{2}[T(x), x]\right)=0, \quad x, y \in R,
$$

whence it follows

$$
[T(x), x] x^{2}+x^{2}[T(x), x]=0, \quad x \in R .
$$

Putting $y x$ for $y$ in (16) we obtain

$$
x^{3} y x[T(x), x]-x y x^{2}[T(x), x] x=0, \quad x, y \in R .
$$


Putting $[T(x), x] y$ for $y$ in the above relation one obtains

(20) $x^{3}[T(x), x] y x[T(x), x]-x[T(x), x] y x^{2}[T(x), x] x=0, \quad x, y \in R$.

Using Lemma the relation (20) turns into

$$
\left(x^{3}[T(x), x]-x^{2}[T(x), x] x\right) y x[T(x), x]=0, \quad x, y \in R .
$$

Putting first $y x^{2}$ for $y$ in the above relation we obtain

$$
\left(x^{3}[T(x), x]-x^{2}[T(x), x] x\right) y x^{3}[T(x), x]=0, \quad x, y \in R .
$$

On the other hand putting $y x$ for $y$ in (21) and right multiplication of this relation by $x$ gives

$$
\left(x^{3}[T(x), x]-x^{2}[T(x), x] x\right) y x^{2}[T(x), x] x=0, \quad x, y \in R .
$$

Subtracting (23) from (22) one obtains

$$
\left(x^{3}[T(x), x]-x^{2}[T(x), x] x\right) y\left(x^{3}[T(x), x]-x^{2}[T(x), x] x\right)=0,
$$

for $x, y \in R$, whence it follows

$$
x^{3}[T(x), x]-x^{2}[T(x), x] x=0, \quad x \in R .
$$

Right multiplication of (18) by $x$ gives

$$
[T(x), x] x^{3}+x^{2}[T(x), x] x=0, \quad x \in R .
$$

According to (24) one can replace in the above relation $x^{2}[T(x), x] x$ by $x^{3}[T(x), x]$. We have therefore

$$
[T(x), x] x^{3}+x^{3}[T(x), x]=0, \quad x \in R .
$$

Left multiplication of (19) by $[T(x), x]$ gives

$$
[T(x), x] x^{3} y x[T(x), x]-[T(x), x] x y x^{2}[T(x), x] x=0, \quad x, y \in R .
$$

Adding relations (20) and (27) and using (26) we obtain

$$
([T(x), x] x+x[T(x), x]) y x^{2}[T(x), x] x=0, \quad x, y \in R .
$$

Using (24) one obtains from the above relation

$$
([T(x), x] x+x[T(x), x]) y x^{3}[T(x), x]=0, \quad x, y \in R .
$$

Left multiplication of (29) by $x^{2}$ gives

$$
\left(x^{2}[T(x), x] x+x^{3}[T(x), x]\right) y x^{3}[T(x), x]=0, \quad x, y \in R .
$$

According to (24) one can replace $x^{2}[T(x), x] x$ by $x^{3}[T(x), x]$ in the above relation. Thus we have

$$
x^{3}[T(x), x] y x^{3}[T(x), x]=0, \quad x, y \in R,
$$

whence it follows

$$
x^{3}[T(x), x]=0, \quad x \in R .
$$


Because of (26) we also have

$$
[T(x), x] x^{3}=0, \quad x \in R .
$$

Putting in (16) $[T(x), x] y$ for $y$ we obtain

$$
x^{3}[T(x), x] y[T(x), x]-x[T(x), x] y x[T(x), x] x=0, \quad x, y \in R .
$$

Using (30), the above relation reduces to

$$
x[T(x), x] y x[T(x), x] x=0, \quad x, y \in R .()
$$

Putting $x y$ for $y$ in (33) we obtain

$$
x[T(x), x] x y x[T(x), x] x=0, \quad x, y \in R,
$$

whence it follows

$$
x[T(x), x] x=0, \quad x \in R .
$$

Linearization of (34) leads to

$$
\begin{aligned}
0= & x[T(x), x] y+x[T(x), y] x+x[T(y), x] x+y[T(x), x] x \\
& +x[T(x), y] y+x[T(y), x] y+y[T(x), x] y+x[T(y), y] x \\
& +y[T(x), y] x+y[T(y), x] x+x[T(y), y] y+y[T(x), y] y \\
& +y[T(y), x] y+y[T(y), y] x, \quad x, y \in R .
\end{aligned}
$$

Putting $-x$ for $x$ in the above relation and combining the relation so obtained with (35) one obtains

$$
\begin{aligned}
& x[T(x), y] y+x[T(y), x] y+y[T(x), x] y \\
& +x[T(y), y] x+y[T(x), y] x+y[T(y), x] x=0, \quad x, y \in R .
\end{aligned}
$$

Comparing (35) and (36) we obtain

$$
\begin{aligned}
& x[T(x), x] y+x[T(x), y] x+x[T(y), x] x+y[T(x), x] x \\
& +x[T(y), y] y+y[T(x), y] y+y[T(y), x] y+y[T(y), y] x=0,
\end{aligned}
$$

for $x, y \in R$. Putting $2 x$ for $x$ in the above relation and subtracting the relation so obtained from the above relation multiplied by 8 , we obtain

$$
x[T(y), y] y+y[T(x), y] y+y[T(y), x] y+y[T(y), y] x=0,
$$

for $x, y \in R$. Comparing (37) and (38) one obtains

$$
x[T(x), x] y+x[T(x), y] x+x[T(y), x] x+y[T(x), x] x=0,
$$

for $x, y \in R$. Right multiplication of (39) by $x^{2}[T(x), x]$ and using (30) one obtains

$$
x[T(x), x] y x^{2}[T(x), x]=0, \quad x, y \in R .
$$

Left multiplication of (40) by $x$ gives

$$
x^{2}[T(x), x] y x^{2}[T(x), x]=0, \quad x, y \in R,
$$


whence it follows

$$
x^{2}[T(x), x]=0, \quad x \in R .
$$

Because of (18) we also have

$$
[T(x), x] x^{2}=0, \quad x \in R .
$$

Right multiplication of (39) by $x[T(x), x]$ gives because of (41)

$$
x[T(x), x] y x[T(x), x]=0, \quad x, y \in R,
$$

whence it follows

$$
x[T(x), x]=0, \quad x \in R .
$$

We also have (using (42))

$$
[T(x), x] x=0, \quad x \in R .
$$

From the above relation one obtains (see how the relation (39) was obtained from (34))

$$
[T(x), x] y+[T(x), y] x+[T(y), x] x=0, \quad x, y \in R .
$$

Right multiplication of (45) by $[T(x), x]$ gives because of (43)

$$
[T(x), x] y[T(x), x]=0, x, y \in R,
$$

whence it follows

$$
[T(x), x]=0, \quad x \in R .
$$

Our next task is to prove the relation

$$
T(x y+y x)=T(y) x+x T(y), \quad x, y \in R .
$$

In order to prove the above relation we need the relations below

$$
x A(x, y) x=0, \quad x, y \in R
$$

and

$$
[A(x, y), x]=0, \quad x, y \in R,
$$

where $A(x, y)$ stands for $T(x y+y x)-T(y) x-x T(y)$. Let us first prove the relation (48). Writing the relation (5) with the sign mentioned above gives

$$
A(x) y x+x y B(x)-x A(x, y) x=0, \quad x, y \in R .
$$

Linearization of the above relation and using (5) gives

$$
\begin{aligned}
& A(z) y x+A(x) y z+x y B(z)+z y B(x)-x A(x, y) z-x A(z, y) x \\
& -z A(x, y) x-z A(z, y) x-z A(x, y) z-x A(z, y) z=0, \quad x, y, z \in R .
\end{aligned}
$$

Putting $-x$ for $x$ in the above relation and adding the relation so obtained with the above relation one obtains

$$
A(x) y z+z y B(x)-x A(x, y) z-x A(z, y) x-z A(x, y) x=0,
$$


for $x, y, z \in R$. In particular for $z=x$ and using (5) the relation (50) reduces to (48). The linearization of (46) gives

$$
[T(x), y]+[T(y), x]=0, \quad x, y \in R .
$$

Putting $x y+y x$ for $y$ in (51) and using (46) one obtains

$$
x[T(x), y]+[T(x), y] x+[T(x y+y x), x]=0, \quad x, y \in R .
$$

According to (51) one can replace in the above relation $[T(x), y]$ by $-[T(y), x]$. Thus we have

$$
[T(x y+y x), x]-x[T(y), x]-[T(y), x] x=0, \quad x, y \in R,
$$

which can be written in the form

$$
[T(x y+y x)-T(y) x-x T(y), x]=0, \quad x, y \in R .
$$

The proof of relation (49) is therefore complete. From the relation (48) one obtains (see how (50) was obtained from its previous relation)

$$
x A(x, y) z+x A(z, y) x+z A(x, y) x=0, \quad x, y, z \in R .
$$

Right multiplication of the above relation by $A(x, y) x$ and using (48) gives

$$
x A(x, y) z A(x, y) x=0, \quad x, y, z \in R .
$$

Because of (49) the above relation can be written as

$$
x A(x, y) z x A(x, y)=0, \quad x, y, z \in R,
$$

which implies

$$
x A(x, y)=0, \quad x, y \in R .
$$

Of course, we also have

$$
A(x, y) x=0, \quad x, y \in R .
$$

Linearization of (55) according to $x$ gives

$$
A(x, y) z+A(z, y) x=0, \quad x, y \in R .
$$

Right multiplication of the above relation by $A(x, y)$ gives because of (54)

$$
A(x, y) z A(x, y)=0, \quad x, y, z \in R,
$$

which implies

$$
A(x, y)=0, \quad x, y \in R .
$$

The proof of relation (47) is therefore complete. In particular for $y=x$ this relation reduces to

$$
2 T\left(x^{2}\right)=T(x) x+x T(x), \quad x \in R,
$$

and because of (46) the above relation yielding $T\left(x^{2}\right)=T(x) x$ and $T\left(x^{2}\right)=$ $x T(x)$ for all $x \in R$. According to Theorem 3 it follows that $T$ is left and 
also right centralizer. Now Theorem 2.3.2. in [1] completes the proof of the theorem.

\section{REFERENCES}

[1] K. I. Beidar, W. S. Martindale and A. V. Mikhalev, Rings with generalized identities, Marcel Dekker Inc., New York, 1996.

[2] M. Brešar and J. Vukman, Jordan derivations on prime rings, Bull. Austral. Math. Soc. 37 (1988), 321-322.

[3] M. Brešar, Jordan derivations on semiprime rings, Proc. Amer. Math. Soc. 104 (1988), $1003-1006$.

[4] M. Brešar, Jordan mappings of semiprime rings, Journal of Algebra 127 (1989), 218228.

[5] J. Cusak, Jordan derivations on rings, Proc. Amer. Math. Soc. 53(1975), 321-324.

[6] I. N. Herstein, Jordan derivations of prime rings, Proc. Amer. Math. Soc. 8 (1957), $1104-1119$.

[7] J. Vukman, Centralizers of semiprime rings, Comment. Math. Univ. Carolinae 42 (2001), 237-245.

[8] B. Zalar, On centralizers of semiprime rings, Comment. Math. Univ. Carolinae 32 (1991), 609-614.

J. Vukman

Department of Mathematics

University of Maribor

PEF, Koroška 160, 2000 Maribor

Slovenia

E-mail: joso.vukman@uni-mb.si

I. Kosi-Ulbl

Department of Mathematics

University of Maribor

PEF, Koroška 160, 2000 Maribor

Slovenia

E-mail: irena.kosi@uni-mb.si

Received: 04.10.2002. 